\title{
Network-based Humanoid 'MAHRU' as Ubiquitous Robotic Companion
}

\author{
Bum-Jae You, Doik Kim, Changhwan Kim, Yong-Hwan Oh, Mun-Ho Jeong, Sang-Rok Oh \\ Center for Cognitive Robotics Research, Korea Institute of Science and Technology \\ Seoul, Korea (Tel: +82-2-958-5760; e-mail: ybj@ kist.re.kr)
}

\begin{abstract}
The paper proposes 'Network-based Humanoid', that is, a humanoid endowed with its perception capability and intelligence by an external computer system connected with wireless network. The network-based humanoid is composed of a humanoid test-bed, an internal control system and an external computer system. The internal distributed control system is composed of two parts. One is responsible for motion control of the humanoid following commands from the external computer system while the motion control is done by a CAN-based distributed motor controller. The other is for real-time data transmission of image data, voice data, and sensor data for motion control by wireless network to the external computer system. The external computer system, a network-based distributed control system, processes the transmitted data, decides the final action command for the humanoid, and transmits the action command to the internal control system. A network-based humanoid, whose name is 'MAHRU', is developed successfully. The humanoid can walk using two legs with the maximum speed of $0.9 \mathrm{Km} / \mathrm{h}$. It is noted that the humanoid can recognize faces, gestures of human beings in real-time, three-dimensional objects, and 100 voice words with the help of the external computer system connected through wireless network. And, the humanoid interacts with human beings via a stereo camera, a microphone, and force/torque sensors.
\end{abstract}

\section{INTRODUCTION}

Humanoid robots take much attention since most people hope to develop an intelligent robot mimicking human beings. There have been developed several humanoids such as ASIMO from Honda Co. Ltd. Japan (Yokoi et al., 2001) (Sakagami et al., 2002), Johnnie from TUM Germany (Loffler et al., 2003), HRP from AIST Japan (Kanehiro et al., 2004), QRIO from Sony Co. Ltd. Japan, HOAP from Fujitsu Co. Ltd. Japan, COG from MIT USA, H6 and H7 from AIST and Univ. of Tokyo (Kagami et al., 2001), HUBO from KAIST Korea (Kim et al., 2005), and etc. Most humanoids have embedded controllers for motion control and/or perception inside of their body even though ASIMO tried to use database in an external server. So, it is not easy to enhance perception functions and service contents and more computers are necessary to install and upgrade functions and perception capability inside of robots.

As the market of industrial robots is matured and the growth of the market is saturated, the need for new markets of intelligent robots has been increased. There have been many trials to develop intelligent robots helpful for human beings in daily life. That is, the robot becomes a consumer product and the robot provides useful services to human beings living together with human beings. One representative killer application of a robot is vacuum cleaning and iRobot Co. Ltd. provided one million vacuum cleaning robots to world market for two years and experts in robotics field recognize that a robot can be a good consumer product under mass production. In order to provide such a consumer robot, the price has to be cheap enough while benefits (of users) obtained from the robot has to be enough. Most developments of robots, however, have been focused on the development of core technologies and the final services provided by the robot are not considered deeply. It is almost impossible to reduce the price of a robot by integrating all functions for control, recognition, artificial intelligence, and service contents inside of the robot. Also, it is not easy to provide and enhance various service contents for a robot in case all of the functions are equipped on the robot while the flexibility for software management of a robot is very important. That is, a new business model for service robots is required to open a new market of service robots. Recently, network-based service robots, URC (Ubiquitous Robotic Companion), are proposed as a business model to decrease the cost of a robot by using external ubiquitous computer systems connected through wireless network and by removing high-level computing system inside of robots. One network-based robot is equipped with an internal control system including motion controller, sensor interface, and network interface while external computer systems connected by wireless network provide high-level signal processing and recognition, reasoning and decision, planning, intelligence for thinking and learning, and etc. The internal control system controls motions of the robot, captures sensor information, and transmits the sensor data to an external computer system through wireless network. So, users can buy a cheap robot and use the robot in their home after registration to a communication service including network and various service contents for the robot. In case the user hopes to use other or more services, the users can buy the 
contents as if they purchase an additive service in cellular phones.

The paper proposes a network-based humanoid, MAHRU, developed successfully by following the concept of networkbased intelligent service robots, URC. Its whole degree-offreedom is 35 while the height and weight are $150 \mathrm{~cm}$ and 67 $\mathrm{Kg}$, respectively. They are equipped with sensors including a stereo camera system, a microphone, force/torque sensors, and a three-dimensional pose sensor. The humanoids can walk using two legs with the maximum speed of $0.9 \mathrm{Km} / \mathrm{h}$. It is noted that the network-based humanoid can recognize faces, gestures of human beings in real-time, threedimensional objects, and 100 voice words by using an external computer system networked by wireless LAN with the internal control system while integrated by CORBA middleware. The overall system is developed and examined successfully via experiments.

The paper is organized as follows. Section 2 introduces the architecture for overall control system including an internal control system and an external control system while section 3 shows dynamic walking mechanism for our humanoid. Section 4 introduces network-based intelligence to recognize human beings, gesture, voice, three-dimensional objects while section 5 shows the developed humanoids. Finally, the paper is concluded in section 6 .

\section{NETWORK-BASED CONTROL SYSTEM}

The whole control system is composed of an internal control system, an external computer system - to provide perception, human-friendly interaction, intelligence, and service contents for human beings - and a remote controller for a networkbased humanoid as shown in Fig. 1.
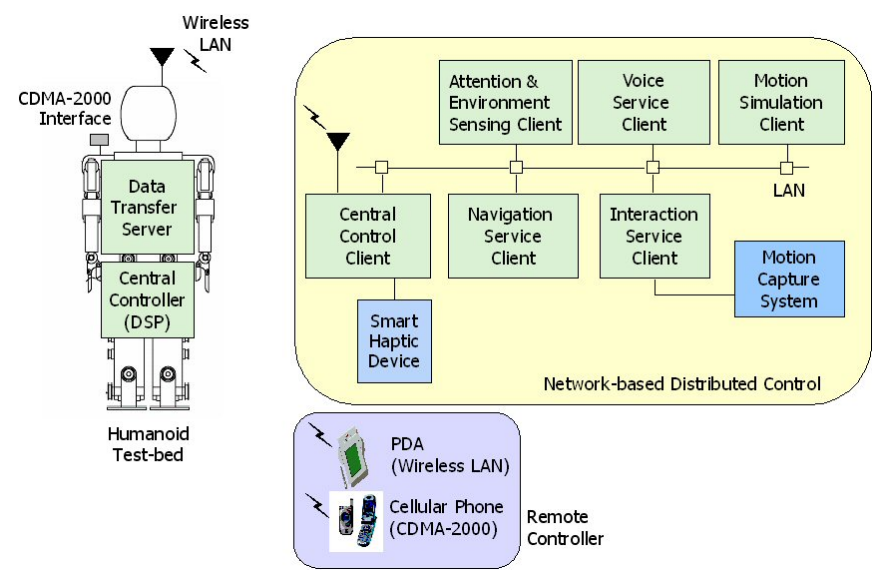

Fig. 1. Network-based Control System

The internal control system is responsible for motor control for each joint, motion control for whole body control, dynamic biped walking and human-like motion of upper body, and real-time transmission of image data, voice data, force/torque data, and motion data for each joint from various sensors. The internal control system is shown in Fig. 2.
Embedded controllers are integrated by CAN (Controller Area Network) whose maximum speed is $1 \mathrm{Mhz}$, and control frequency for walking and whole body control of the humanoid is $400 \mathrm{~Hz}$.

Fig. 3 shows a joint controller developed using TMS320VC33 DSP equipped with motion control algorithm and communication via CAN while a motion coordination controller connected with a number of joint controllers via CAN is shown in Fig. 4. The joint controller controls velocity and position of each joint with $1 \mathrm{KHz}$ rate while the coordination controller includes algorithms for dynamic walking and whole-body motion control of our humanoid. An industrial PC is used for real-time data transfer of images from a Bumble-Bee stereo camera, a microphone, and other sensor information for motion.

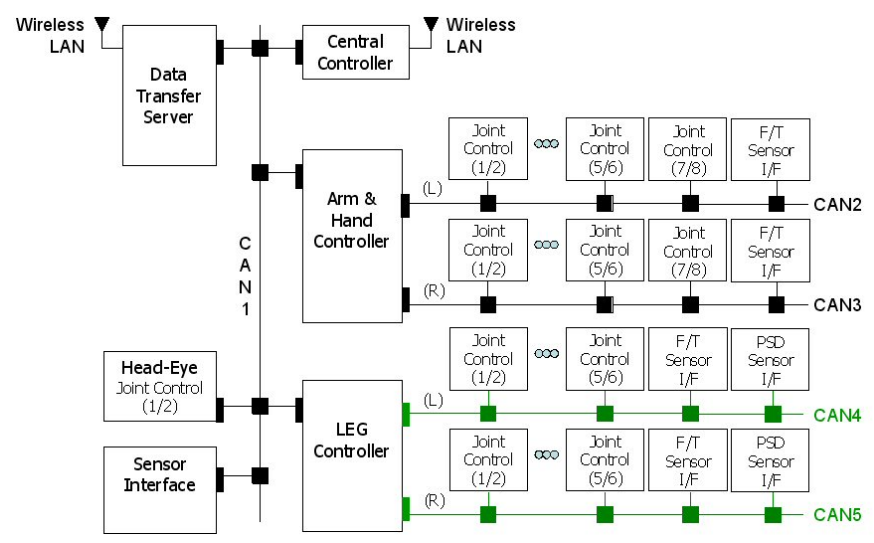

Fig. 2. Internal Control System
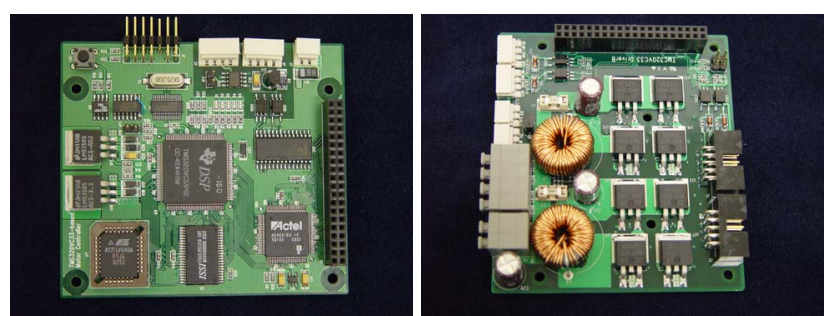

Fig. 3. CAN-based Joint Controller \& Driver

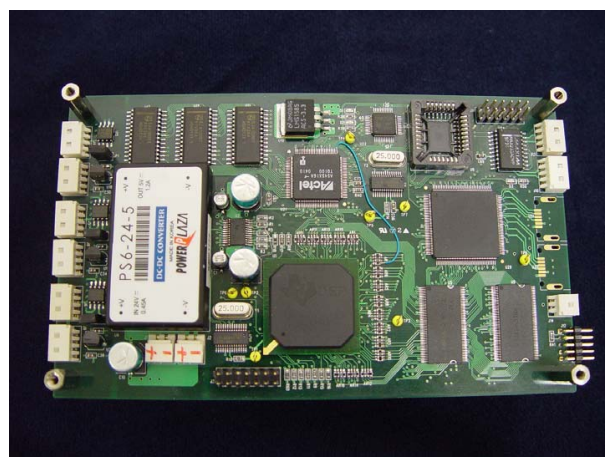

Fig. 4. Motion Coordination Controller

The external computer system is composed of several service clients to provide perception, human-friendly interaction, 
intelligence, and service contents for human beings. The external computer system can provide same services for multiple network-based humanoids by using multi-tasking capability. All clients are integrated with CORBA middleware while software components in each client are modularized by using CORBA interface. Central control client is responsible for real-time data transmission and action command generation after reasoning for commands from other service clients. Also, it selects proper clients to provide a service to a network-based humanoid and sends requests for service to selected clients. The central client is the final decision maker of the external computer system. Attention and environment sensing client tracks and recognizes faces and bodies of human beings, recognizes three-dimensional objects, makes a three-dimensional model of environments. Interaction client recognizes still and continuous gestures of human beings and provides an interaction framework between the humanoid and human beings. Navigation client decides the current pose - position and orientation - of the humanoid in environments, detects obstacles, provides paths to avoid obstacles and to go to a goal point. Voice service client recognizes human voice in word and syntheses human voices while motion capture system provides natural motion profiles for the humanoid captured from human motions and optimized for the robot.

\section{WHOLE BODY CONTROL}

\subsection{Dynamic Biped Walking}

Stable biped walking mechanism is essential in humanoids, as shown in Fig. 5, and there have been proposed many researches on motion control of humanoids such as walking control, running control, and whole body coordination (Choi et al., 2005) (Grizzle et al., 2003) (Hirai et al., 1998) (Kajita et al., 2001) (Kajita et al., 2002) (Kajita et al., 2003) (Kim et al., 2004). We develop a kinematic resolution method of CoM(center of mass) Jacobian with embedded (walking or dancing) motion that offers the ability of whole body control to the humanoid. For example, if the humanoid stretches two arms forward, then the position of CoM of the humanoid moves forward and its ZMP(zero momentum point) swings back and forth. In this case, our kinematic resolution method offers configurations of supporting limbs which are calculated automatically to maintain the position of CoM fixed at a constant point.

Since legs of humanoids have high degree-of-freedoms for human-like natural walking, it is not easy to design a controller and to analyze the stability. So, we simplify walking-related dynamics of humanoids as the equation of motion for a point mass at CoM and propose a rolling sphere model for dynamic walking shown in Fig. 6. And, we propose the walking controller shown in Fig. 7 for dynamic walking by using the rolling sphere model for biped walking. The ZMP planner and CoM planner generate desired trajectories while the ZMP controller and CoM controller control humanoids to minimize errors between real ZMP and desired ZMP and between real CoM and desired CoM simultaneously.

To implement robot walking, first of all, the stepping positions on the ground and the supporting phases are predetermined as shown in Fig. 8. In this figure, the stepping positions are generally represented as periodic functions and the supporting phases (double supporting and single supporting) are used in moving the ZMP. In a single supporting phase, the ZMP should stay in the sole of supporting leg while the shifting leg is making a step. In a double supporting phase, the ZMP should be moved to the sole of shifting leg. These procedures should be repeated to make stable robot walking.

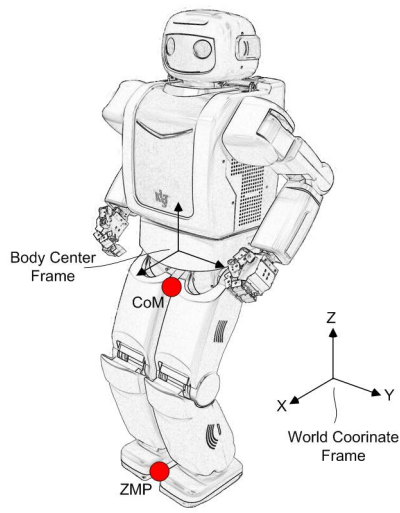

Fig. 5. Coordinate frame

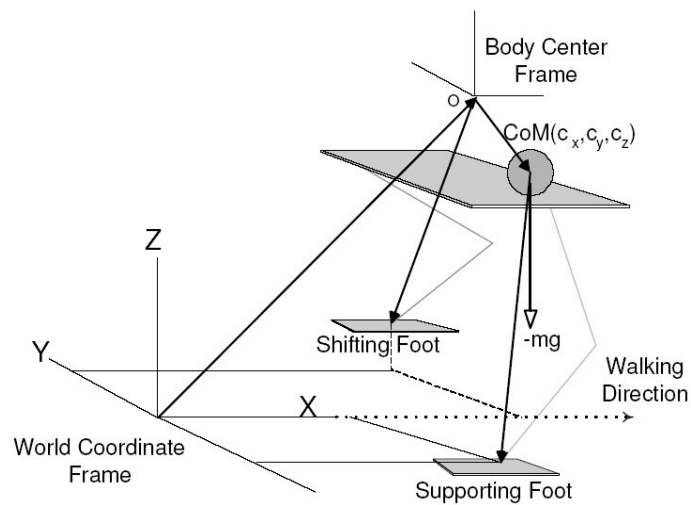

Fig. 6. Rolling sphere model for dynamic walking

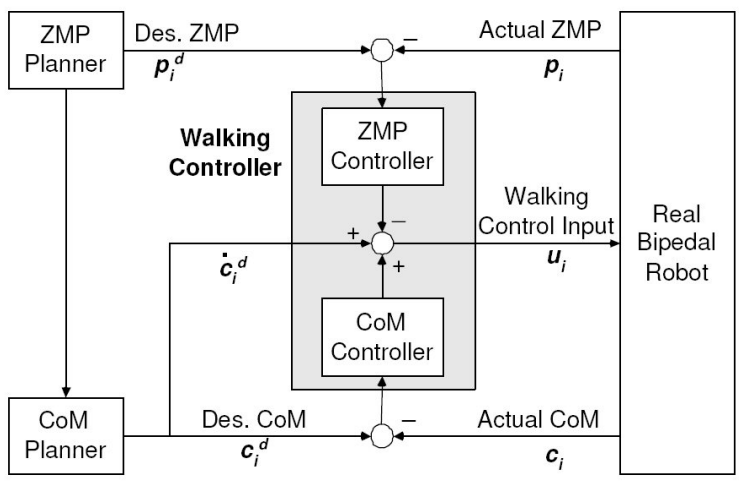

Fig. 7. Walking Controller 
Also, the desired trajectory of CoM should be derived from the desired ZMP in Fig. 8. The takeoff time, landing time and step length of the shifting leg are all determined dependently upon the supporting phases and ZMP planning in Fig. 8.

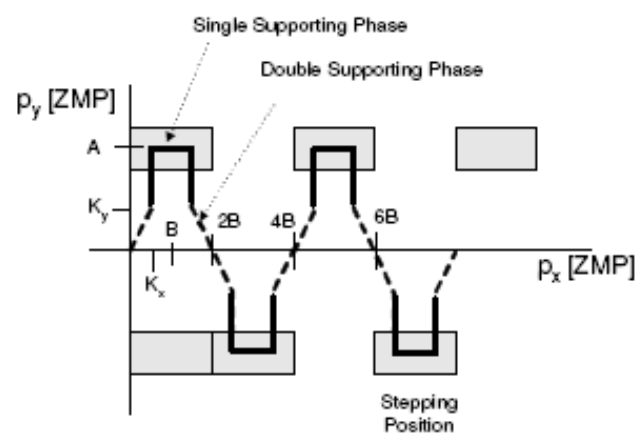

Fig. 8. Desired ZMP Trajectory

\subsection{Motion Imitation of Human Upper Body}

Human motions performed by a person (an actor) are first recorded by Hawk Digital System, commercially available from Motion Analysis Inc., in terms of the time trajectories of all the markers attached on the actor's body. A geometric scaling rule is then applied to overcome the geometric differences between those two characters. The human motions represented by the marker trajectories are adapted to the geometrically scaled humanoid robot. This adaptation procedure is performed with optimization. The motions of upper body and lower body of the actor are separately considered in the procedure. The motions of upper body consisting of head and trunk are converted into motions available to a humanoid robot by computing relative positions of markers on those bodies. For arm motions, joint position and velocity trajectories are obtained using the present method under the assumption that the joint positions and velocities for robot arms are bounded. We are now, however, under development an adaptation method of human leg motions since the conversion process for lower body motions is different from for the upper body due to the balancing issue.

It is well known that applying captured human arm motions to a humanoid robot may not be achieved easily due to geometric and dynamic differences between a human arm and a humanoid robot arm such as arm length difference, length rate difference of upper and lower arms, fewer DOF of a humanoid robot than those of a human and dynamics capability difference. To resolve these difficulties, an efficient method using optimization and a simpler way to impose limits of joint position and velocity are developed.

To evaluate the developed method one motion of the Korean traditional folk dance Arirang were captured by the motion capture system. For the dance, 41 markers were used for the full body and captured at the rate of $120 \mathrm{~Hz}$ with 8387 frames. The transformed motion is applied for the second version of network-based humanoid, MAHRU-II, that has 5 DOF $(N=$ 5) available for each arm imitated the dance motion as shown in Fig. 9.
The arm motions of dance were imitated well using the proposed method even though the robot has only 5 DOF for an arm. The leg motions were generated by the whole body balancing algorithm in (Kim et al., 2005) to avoid falling down. Indeed the captured dance motion involves several foot steps but those motions were not considered yet. The arm motion is only imitated by the proposed method herein, while the leg motions were generated using the captured pelvis motion of actor for balancing in standing status as seen in Fig. 14. The figure shows dancing during the APEC (AsiaPacific Economic Cooperation) 2005 held in the city of Pusan, Korea.

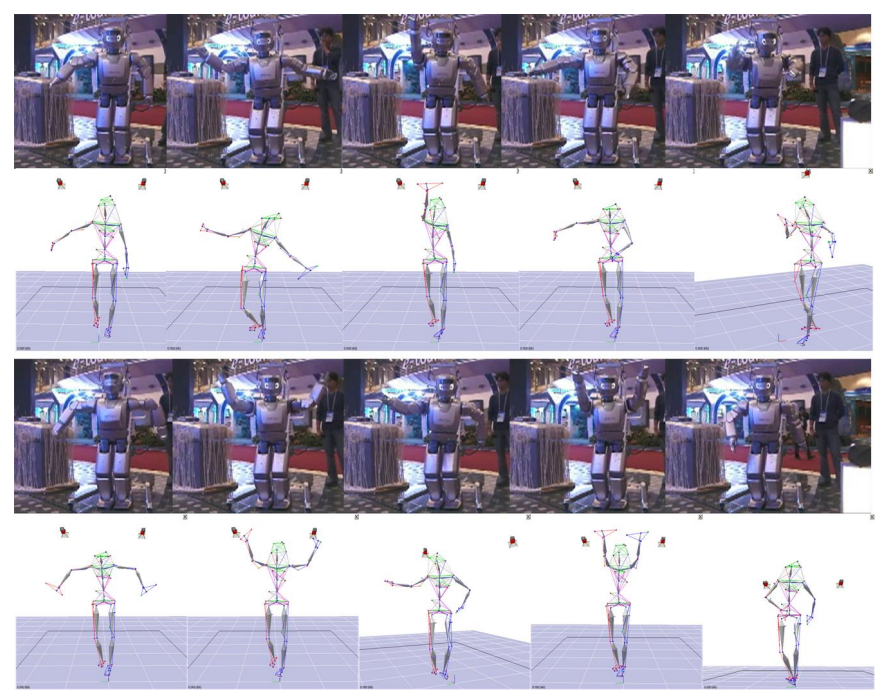

Fig. 9. Imitation of Korean fork dance Arirang

\section{NETWORK-BASED PERCEPTION}

By adopting the network-based architecture, it becomes possible to endow the humanoid with various recognition capabilities for faces, gestures, voices, and three-dimensional objects. All clients for distributed computing are integrated by CORBA middleware while each software module is combined as a functional component in CORBA environment

To apply visual processing capability to robots, visual processing algorithms have to be robust to illumination variation and geometrical variation of objects. For example on face recognition, we have to detect face regions in a robust manner under various illumination conditions as shown in Fig. 10. A robust color model for skin color is used by using a Gaussian model including brightness information of a pixel.
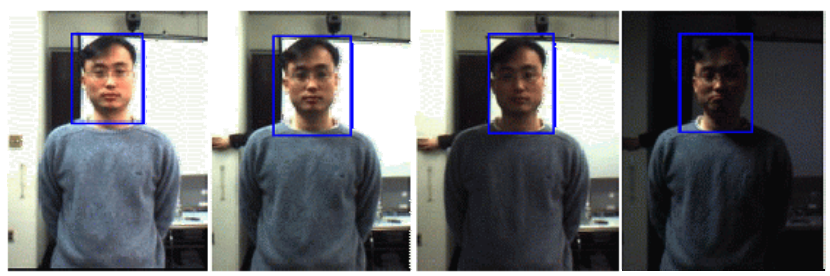

Fig. 10. Robust Face Detection 
Although any camera system is used under the same illuminators, the single-colored objects have different color distributions due to irregular brightness and the viewing geometry of the camera system. Since chrominance tends to be less affected by brightness changes, such chromatic color spaces as the UV plane of the YUV space, the HS plane of the HSI space, and the normalized RG plane of the RGB space have often been used. The scheme ignoring the luminance component suffers from the fact that practically the chromatic color distribution is not invariant enough to disregard the effect of brightness changes. Some adaptation methods using the shape of the color histogram were proposed to cope with the brightness changes, but they still have difficulties in being adapted to the abrupt changes in brightness and the initial detection (segmentation) problem under various brightness conditions.

We extend the idea in (Kim et al., 2003) to achieve a compactly parameterized color model that represent all chromatic color distributions with respect to effective brightness range. Since the proposed Gaussian-cylindrical color model, shown in Fig. 11, can explain the variability with respect to all brightness values, it can provide an effective cue for object tracking against irregular and abrupt changes in brightness. From this point we introduce the mean-shift iteration utilizing the Gaussian-cylindrical color model and a simple and efficient method for finding the optimal bandwidth of the kernel density function since bandwidth selection in the mean-shift algorithm is critical to robust and effective mean-shift iteration. The optimal bandwidth maximizes the lower bound of the log-likelihood of the tracking target. Results are shown in Fig. 12.

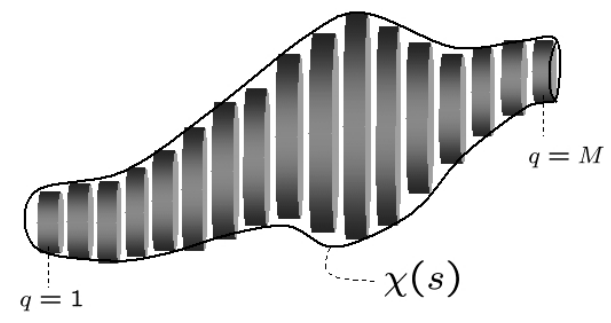

Fig. 11. Gaussian-cylindrical color model

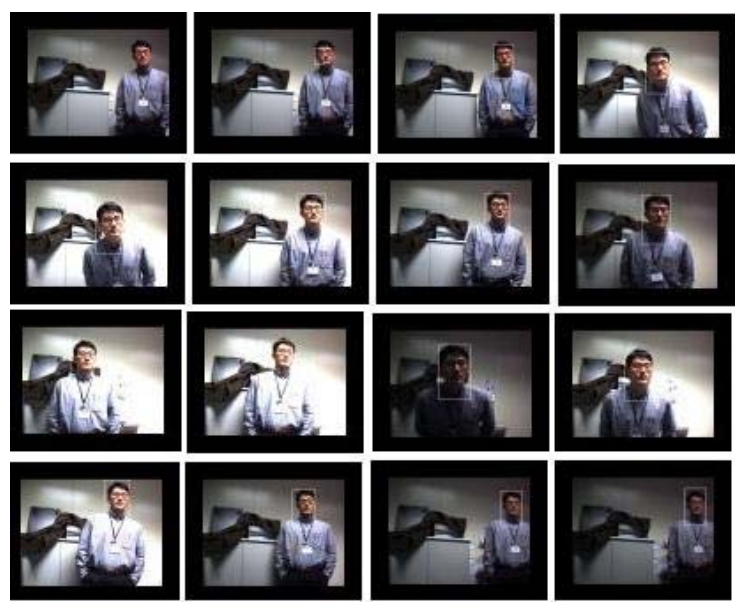

Fig. 12. Face Tracking
Fig. 13 shows an experimental result for face tracking, detection and recognition. The left figure shows visual tracking and recognition of faces of two persons. The characters on the upper side of the window show names of persons.

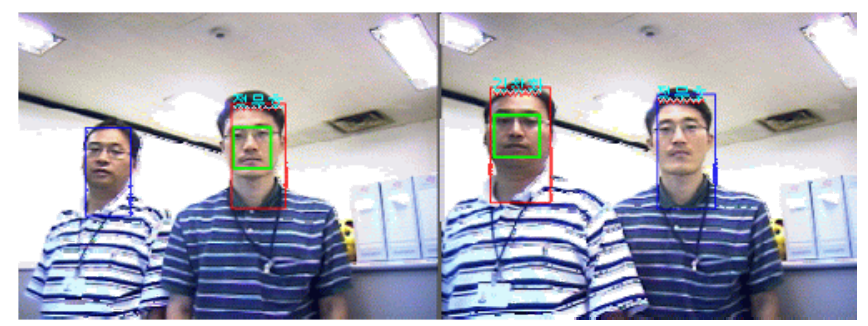

Fig. 13. Face Recognition

Fig. 14 includes examples for gesture recognition by proposing active plane model of feature points grids for human motions based on three-dimensional information of human body. The dots in images show feature points for gesture recognition.
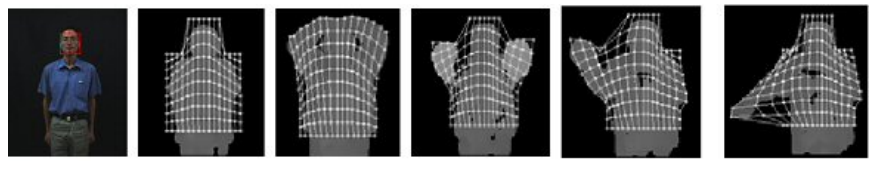

Fig. 14. Gesture recognition

\section{NETWORK-BASED HUMANOID 'MAHRU'}

Network-based humanoid, MAHRU, is developed by combining an humanoid with information technology using the external computer system connected by wireless network as shown in Fig. 15. The humanoid has 35 degree-of-freedom including 6 DOF in each arm, 4 DOF in each hand, 6 DOF in each leg, 2 DOF in each neck, and 1 DOF in each trunk. The height of the humanoid is $150 \mathrm{~cm}$ while the weight is $67 \mathrm{Kg}$. The maximum walking speed is $0.9 \mathrm{Km} / \mathrm{h}$ in planar regions under the assumption that there is no severe variation of the height. The humanoid is equipped with a stereo camera system, a microphone, four force/torque sensors, and a pose sensor. Various recognition capabilities are provided by the help of the external computer system networked by wireless LAN whose maximum network speed is $54 \mathrm{Mbps}$. The external computer system is composed of six PCs whose CPU frequency is $3.2 \mathrm{GHz}$ connected by Gigabit LAN. The frequency of whole body control for the humanoid is $400 \mathrm{~Hz}$ while the frequency of high-level processing is about $12 \mathrm{~Hz}$.

\section{CONCLUSIONS}

In this paper, the concept of network-based humanoid is proposed and network-based humanoids, whose names are MAHRU and AHRA, are developed by integrating humanoids with a networked external computer system through wireless LAN successfully. The humanoid is 
developed following the concept of URC, ubiquitous robotic companion, and the possibility to endow a robot with highlevel perception and/or intelligence is shown by using the external computer system.

High-level intelligence, such as learning, reasoning, planning, evolution, recognition, and etc, will be developed continuously as primary elements for network-based humanoids, and intelligent control architecture will be investigated to control and coordinate multiple humanoids by using efficiently various external ubiquitous clients.
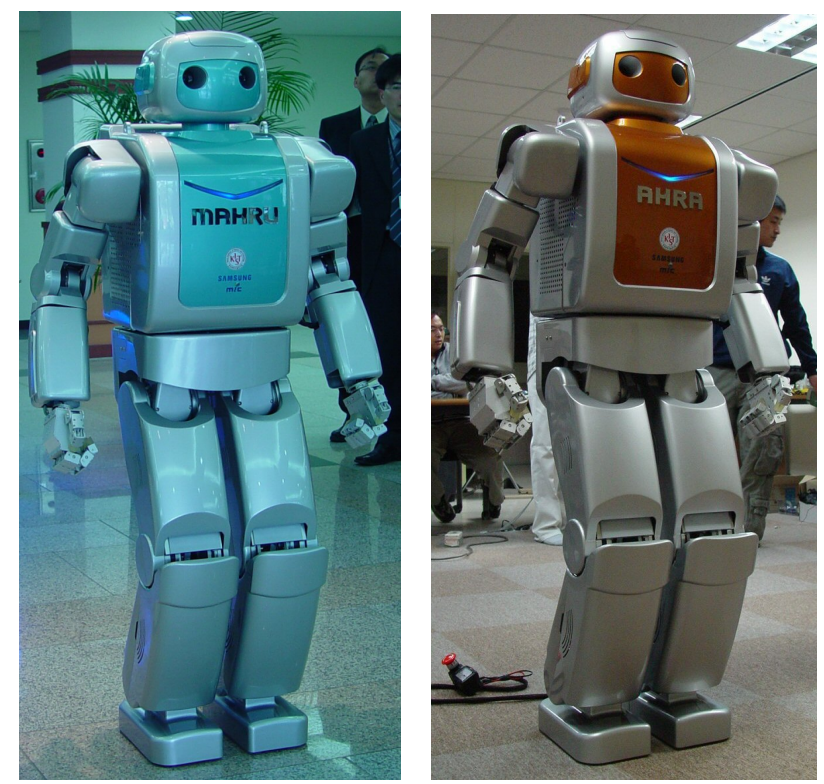

Fig. 15. MAHRU and AHRA

\section{REFERENCES}

K. Yokoi, F. Kanehiro, K. Kaneko, K. Fujiwara, S. Kajita, and H. Hirukawa (2001), "A Honda Humanoid Robot Controlled by AIST Software", Proceedings of the IEEE-RAS International Conference on Humanoid Robots.

Y. Sakagami, R. Watanabe, C. Aoyama, S. Matsunaga, N. Higaki, and K. Fujimura (2002), "The intelligent ASIMO: System Overview and Integration", Proceedings of the IEEE/RSJ International Conference on Intelligent Robots and Systems, pp. 2478-2483.

K. Loffler, M. Gienger, and F. Pfeiffer (2003), "Sensors and Control Concept of Walking 'Johnnie"', International Journal of Robotics Research, vol. 22, no. 3-4, pp. 229239.

F. Kanehiro, H. Hirukawa, and S. Kajita (2004), "OpenHRP: Open Architecture Humanoid Robotics Platform", International Journal of Robotics Research, vol. 22, no. 2, pp. $155-165$.

S. Kagami, K. Nishiwaki, J. Kuffner, K. Okada, Y. Kuniyoshi, M. Inaba, H. Inoue (2001), "Low-level Autonomy of the Humanoid Robots H6 \& H7", Proceedings of International Symposium on Robotics Research, pp. 50-58.
J. -Y. Kim, I. -W. Park, J. Lee, M. -S. Kim, B. -K. Cho, and J. -H. Oh (2005), "System Design and Dynamic Walking of Humanoid Robot KHR-2", Proceedings of IEEE International Conference on Robotics and Automation, pp. 1443-1448.

Y. Choi, B. -J. You, and S. -R. Oh (2005), "On the stability of indirect ZMP controller for biped robot systems", Proceedings of IEEE/RSJ International Conference on Intelligent Robots and Systems, pp.1966-1971.

J. W. Grizzle, E. R. Westervelt, and C. C. de Wit (2003), "Event-based PI Control of a Under-actuated Biped Walker", Proceedings of IEEE International Conference on Decisions and Control, pp.3091-3096.

K. Hirai, M. Hirose, Y. Hakikawa, and T. Takenaka (1998), "The Development of Honda Humanoid Robot", Proceedings of IEEE International Conference on Robotics and Automation, pp.1321-1326.

S. Kajita, M. Saigo, and K. Tanie (2001), "Balancing a Humanoid Robot using Backdrive concerned Torque Control and Direct Angular Momentum Feedback", Proceedings of IEEE International Conference on Robotics and Automation, pp.3376-3382.

S. Kajita, F. Kanehiro, K. Kaneko, K. Fujiwara, K. Yokoi, and H. Hirukawa (2002), "A Real-time Pattern Generator for Biped Walking", Proceedings of IEEE International Conference on Robotics and Automation, pp.31-37.

S. Kajita, F. Kanehiro, K. Kaneko, K. Fujiwara, K. Harada, K. Yokoi, and H. Hirukawa (2003), "A Real-time Pattern Generation by using Preview Control of Zero-moment Point", Proceedings of IEEE International Conference on Robotics and Automation, pp.1620-1626.

J. H. Kim and J. H. Oh (2004), "Walking Control of the Humanoid Platform KHR-1 based on Torque Feedback Control", Proceedings of IEEE International Conference on Robotics and Automation, pp.623-628.

D. Kim, Y. Choi, and C. Kim (2005), "Motion-embedded cog jacobian for a real-time humanoid motion generation", The Second International Conference on Informatics in Control, Automation and Robotics, volume 2, pages 5561, Barcelona, Spain.

C. H. Kim, B.-J. You, H. Kim, and S.-R. Oh (2003), “A Robust Color Segmentation Technique for Tangible Space", Proceedings of XVth Triennial Congress of International Ergonomics Association, Seoul, Korea. 